\title{
Knowing beans: human mirror mechanisms revealed through motor adaptation
}

\section{Arthur M. Glenberg', ${ }^{1}$, Gabriel Lopez-Mobilia', Michael McBeath ${ }^{1}$, Michael Toma $^{1}$, Marc Sato ${ }^{3}$ and Luigi Cattaneo ${ }^{4}$}

\author{
Department of Psychology, Arizona State University, Tempe, AZ, USA \\ 2 Department of Psychology, University of Wisconsin-Madison, Madison, WI, USA \\ ${ }^{3}$ GIPSA-LAB, CNRS UMR 5216, Grenoble University, Grenoble, France \\ ${ }^{4}$ Neuroimaging Laboratories, Center for Mind/Brain Sciences, University of Trento, Mattarello, Italy
}

Edited by:

Stephen A. Engel, University of

Minnesota, USA

Reviewed by:

Jonas Kaplan, University of Southern California, USA

Pascal Molenberghs, University of

Queensland, Australia

${ }^{*}$ Correspondence:

Arthur M. Glenberg, Department of

Psychology, Arizona State University,

Tempe, AZ 85287-1104, USA.

e-mail: glenberg@asu.edu
Human mirror mechanisms (MMs) respond during both performed and observed action and appear to underlie action goal recognition. We introduce a behavioral procedure for discovering and clarifying functional MM properties: blindfolded participants repeatedly move beans either toward or away from themselves to induce motor adaptation. Then, the bias for perceiving direction of ambiguous visual movement in depth is measured. Bias is affected by (a) number of beans moved, (b) movement direction, and (c) similarity of the visual stimulus to the hand used to move beans. This cross-modal adaptation pattern supports both the validity of human MMs and functionality of our testing instrument. We also discuss related work that extends the motor adaptation paradigm to investigate contributions of MMs to speech perception and language comprehension.

Keywords: mirror mechanisms, action recognition, motor adaptation, visual perception

\section{INTRODUCTION}

Mirror neurons (or mirror mechanisms, MMs) are active both when a macaque monkey takes action and when the animal observes others perform similar actions (Rizzolatti and Craighero, 2004). Thus, MMs appear to function in action goal recognition and may underlie social behaviors such as cooperation (Kilner et al., 2006) and social communication (Glenberg, 2007; Rizzolatti and Craighero, 2007). In humans, the existence and function of MMs is controversial in part because brain imaging alone, the technique often used to test for MMs, cannot determine that the same neurons contribute to both action and perception, nor can these data reliably determine function (Dinstein et al., 2008; Hickok, 2009). Here we demonstrate a behavioral method using motor adaptation that produces convincing evidence for human MM existence and function. Blindfolded participants repeatedly transferred objects either toward or away from the body, thus inducing adaptation. Then, we measured the threshold to detect movement of a visual stimulus in depth as toward or away. Consistent with the claim that we tapped a MM, the threshold was affected by number of objects transferred, direction of transfer, and similarity of the visual stimulus to the effector used to transfer the objects. Related studies have shown that similar forms of motor adaptation affect speech perception and language understanding (Glenberg et al., 2008; Sato et al., 2008). Thus, the adaptation procedure can be used to study human MMs function in multiple systems.

Investigation of area F5 of the macaque premotor cortex produced the first identification of mirror neurons (Di Pellegrino et al., 1992). The signature finding, based on single-cell recording, is that the same motor neuron fires both during production of an action and during perception of the same action (about onethird of mirror neurons) or similar actions, that is, actions with the same goal (about two-thirds of mirror neurons). About 17\% of mirror neurons have auditory, as well as visual and motor properties (Kohler et al., 2002).

Several experiments with macaques suggest a role for mirror neurons in action goal recognition. For example, a mirror neuron will be active when an action on an object is completed behind a barrier, but not when the animal knows that there is no object behind the barrier (Umiltà et al., 2001). Also, a mirror neuron will fire when pliers operated by the animal close on a target, and the same neuron is active when the pliers operate by a hand-closing or a hand-opening movement (Umiltà et al., 2008). Thus, it is the goal that counts, not the specific movements for achieving the goal.

Because of the relative scarcity of single cell recording in humans (but see Mukamel et al., 2010), much of the human mirror neuron literature uses functional magnetic resonance imaging (fMRI). In this case, the operation of MMs is inferred when activity in a particular cortical area (as reflected in the BOLD signal) is similar during action recognition and action production, and when the cortical area is a likely homolog of an area in macaque cortex in which mirror neurons have been identified. Using this or a similar logic, it appears that the human MMs respond more to actions that the perceiver can perform than to actions the perceiver is familiar with through vision alone (Calvo-Merino et al., 2006); MMs are more sensitive to social actions than similar non-social actions (Kilner et al., 2006); MMs respond to both visually perceived actions and the linguistic description of actions (Tettamanti et al., 2005); MMs play a role in speech perception (D'Ausilio et al., 2009); activity in MMs is positively correlated with empathy (Gazzola et al., 2006) and negatively correlated with autistic behaviors (Dapretto et al., 2006).

Nonetheless, there are reasons to be skeptical. Namely, fMRI data cannot clearly demonstrate that the same neurons are contributing to both action production and action recognition, only that a 
particular area of cortex (which may summate activity in hundreds of thousands of neurons with different functions) is active for both (Dinstein et al., 2008). Note that the same criticism applies to many studies using transcranial magnetic stimulation (TMS) given its limited spatial resolution. Although TMS will disrupt neural processing at a particular location, it is unclear if the disruption is due to disruption of multi-modal mirror neurons or to disruption of different unimodal neurons in the same location.

Dinstein et al. (2008) suggest a solution for fMRI investigations of MMs: adaptation paradigms. Repeated presentation of a visual stimulus adapts the BOLD signal. Then, if that previous visual presentation modulates the BOLD signal produced during action production, this modulation provides strong evidence in support of a MM. Unfortunately, several experiments reviewed by Dinstein et al. (2008) failed to find the cross-task adaptation. A more recent study does demonstrate cross-task adaptation in the BOLD signal (Chong et al., 2008). However, as discussed in Dinstein (2008), the location of the adapted BOLD signal was unexpected on the basis of previous work putatively supporting a human MM. Also, Lingnau et al. (2009) report asymmetric adaptation (a visual stimulus adapts the BOLD signal during production but the reverse is not found) which they interpret as more consistent with a cognitive-level priming mechanism than a neural-level MM. In contrast, Kilner et al. (2009) do report symmetric cross modal adaptation (from observation to execution and the reverse) in human inferior frontal gyrus (IFG), which is a location that has been associated with mirror neurons in the macaque. It is likely that Kilner et al. (2009) were successful because (a) they focused on IFG rather than whole brain imaging thus obtaining more observations in this critical area for mirror neurons, and (b) they used tasks with clear action goals rather than mimed movements. Monkey mirror neurons appear to be more sensitive to movements with clear goals than those without (e.g., Umiltà et al., 2001), and the same might be true in humans.

Thus, cross-modal adaptation has proved itself to be a potentially effective technique for investigating human MMs. However, the demonstration of cross-modal adaptation using fMRI remains correlational. That is, whether or not this adaptation has a causal effect on cognition or behavior is unknown. The technique that we describe next uses a behavioral version of cross-modal adaptation that allows for causal inferences.

The behavioral method takes advantage of the general property of cortical neurons that they adapt with use, along with the following three properties of mirror neurons: (a) they are multi-modal, sensorimotor neurons, (b) they are specific to particular actions and goals, and (c) they differentially couple to biological stimuli.

In the work reported here, blindfolded participants transferred cannellini beans, one at a time, from a source container with a wide mouth to a target container with a narrow mouth. With the source container near to the participant and the target container $25 \mathrm{~cm}$ away, repeated movements putatively adapt away-action control, and with the locations of the containers reversed, repeated movements adapt toward-action control. Half the participants moved beans in each direction, and orthogonally, participants moved 15, 45,135 , or 405 beans. Note that moving beans into a target container requires movements both toward the container and away from the container (to retrieve the next bean). Because we identify to the participant the goal ("move the beans one at a time into the target container"), and because mirror neurons are more sensitive to goals than literal movements, we are able to unambiguously classify the direction of movement in regard to location of the target container relative to the participant.

Immediately following bean movement, we measured the threshold to detect ambiguous movement in depth as toward or away (Lewis and McBeath, 2004). The participant viewed a screen tiled with repetitions of a stimulus appearing to recede in depth (see Figure 1). After $1 \mathrm{~s}$, the computer display presented a simulation of the stimuli moving in depth a particular percentage of the inter-tile distance, and the participant responded as to whether the tiles appeared to move toward or away. On subsequent presentations, the percentage was adjusted until the participant could not reliably determine the direction of movement. We will refer to the percentage of inter-tile shift for which the direction of movement cannot be reliably determined as the threshold. The question of interest is whether direction of bean movement differentially shifts the threshold. Namely, if the threshold increases after motor adaptation, then participants need more information to see a shift in the toward direction, and if the threshold decreases after motor adaptation, then participants need less information to see a shift in the toward direction. Lewis and McBeath (2004) have demonstrated that the threshold is susceptible to various biases.

\section{MATERIALS AND METHODS}

The 134 participants were right-handed students enrolled in Introductory Psychology classes at Arizona State University who participated to fulfill a course research requirement. All procedures were approved by the local institutional review board. Participants were randomly assigned to one of eight conditions formed by combining two levels of bean movement Direction (Toward and Away) with four levels of Number of beans (15, 45, 135, and 405).

Bean task practice consisted of placing the left hand on the target container (which was attached to the desk) and using the right hand to move two beans from the source container to the target container. Then, participants were blindfolded and moved the remaining beans.

After completing the bean task, participants moved in front of a computer monitor. The distance between the monitor and the participant was $36 \mathrm{~cm}$, and that distance was maintained by a chinrest. Also, the angle of the monitor to the desk was maintained at $110^{\circ}$ (these parameters can affect the perceived direction of motion).

The threshold task was modeled after Lewis and McBeath (2004), but instead of the staircase procedure, we used the MOBS procedure (Tyrrell and Owens, 1988) which reduces measurement error. Each trial began with the presentation, for $1 \mathrm{~s}$, of one of the tile displays in Figure 1. Next, a new display was presented to simulate movement in depth. The movement was simulated by generating a new image based on (a) movement of the images a particular percentage of the inter-tile distance in the horizontal plane, and (b) the geometric projection of this image onto the plane of the computer screen, as if the screen were a window through which one observed the movement of the tiles in the horizontal plane. Because new (correctly scaled) tiles appeared at the tops and bottoms of the displays, the movement was ambiguous. That is, the same display might be produced by a small percentage shift in one direction or a larger percentage shift in the opposite direction. Nonetheless, a large percentage (e.g., 80\%) 


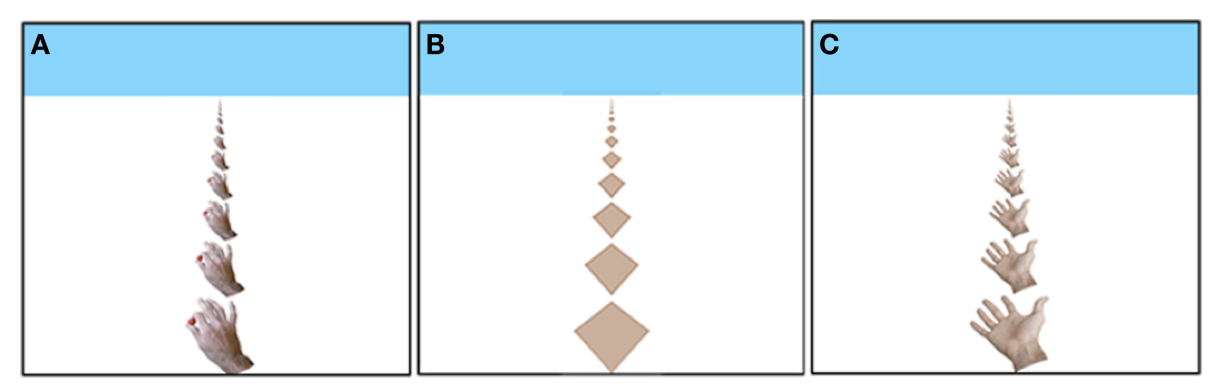

FIGURE 1 |The Hand stimulus tiling (A); the Diamond stimulus tiling (B); the Open Hand stimulus tiling (C).

reliably produces perceived movement toward the observer, and a small percentage (e.g., 20\%) reliably produces perceived movement away from the observer. After each shifted display, the participant responded by pressing the up-arrow key on the computer keyboard to indicate that the shift was perceived as away, or the participant pressed the down-arrow key to indicate that the shift was perceived as toward the participant.

The threshold procedure began by presenting $80 \%$ and $10 \%$ shifts quasi-randomly assigned to the three stimuli so that each percentage was used at least once. After each response, the MOBS procedure was used to compute a percentage shift for the next trial. In brief, the percent inter-tile distance in the shift between the two displays for the next trial was the average of estimated upper (initially $80 \%$ ) and lower (initially 10\%) boundaries of the threshold, and the two boundaries were adjusted after every response. Separate boundaries were maintained for the Hand, Open Hand, and Diamond stimuli, although the boundaries were reset and thresholds determined simultaneously for the three stimuli. That is, a participant responded to one Hand stimulus shift, one Open Hand stimulus shift, and one Diamond shift (in an interleaved random order determined for each participant) with the boundaries adjusted after each response. These three shifts were followed by another three, and so on, until the thresholds were determined for all three stimuli. A threshold was determined as the average of the upper and lower boundaries when a) at least seven reversals in the direction of perceived movement occurred, and (b) the final inter-boundary distance was less than $5 \%$ of the starting boundary distances ( $10 \%$ and $80 \%$ shifts). Then, the boundaries were reset to the starting values and a second threshold determined for all three stimuli. The data entered into the statistical analyses consisted of the average of these two thresholds.

\section{RESULTS}

Adaptation produced by the bean task is only expected to last a few minutes (Classen et al., 1998). Similarly, Cattaneo et al. (submitted) used a short-term ( $1 \mathrm{~min}$ ) motor adaptation procedure and found that the effects of adaptation on action recognition persisted for only $30 \mathrm{~s}$. Consequently, we eliminated from the analyses data from participants who took longer than $7 \mathrm{~min}$ (about half the time spent in the bean task) to complete the threshold determination $(24 \%$ of the participants).

The threshold data are displayed in Figure 2. As the number of Toward and Away bean movements increased, there was an increasingly large difference between the thresholds. This interaction

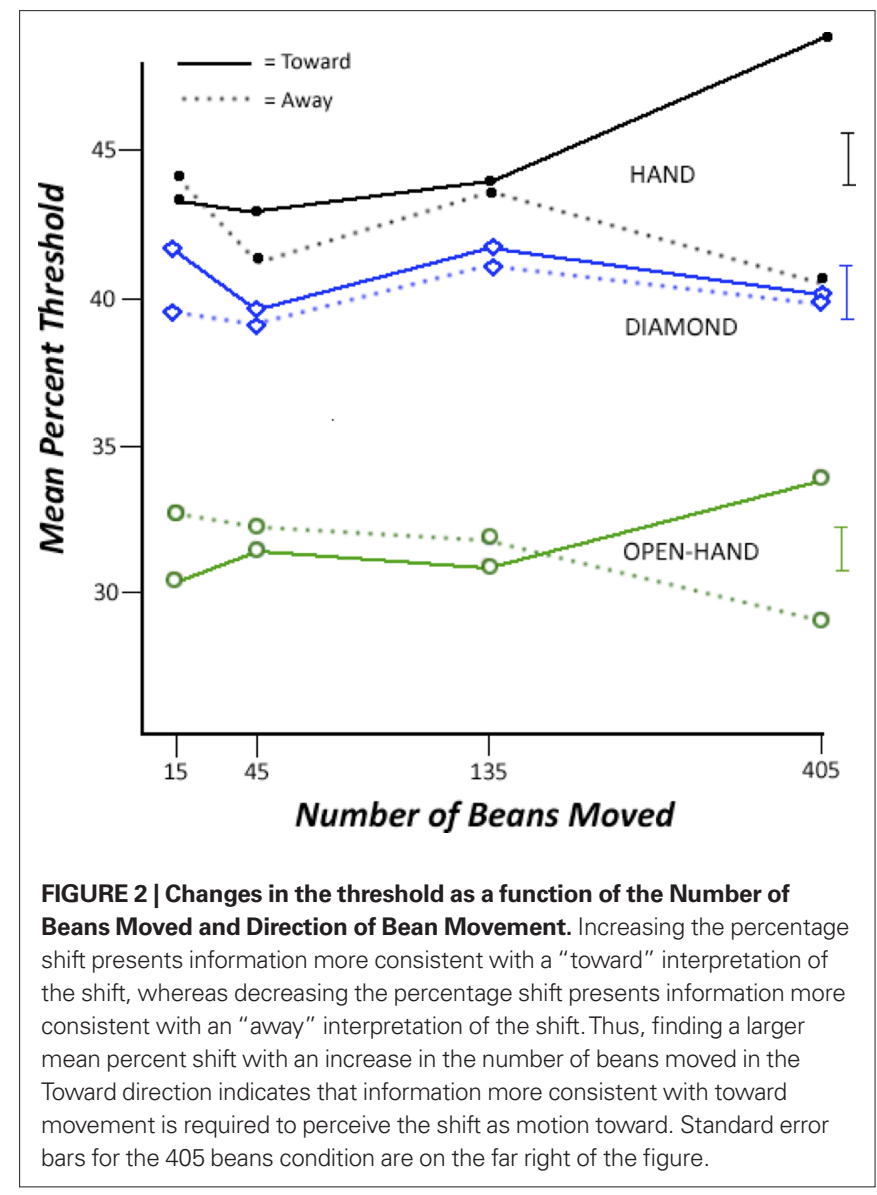

effect was modulated by the biological verisimilitude of the visual stimulus to the hand that had literally moved the beans. That is, the interaction was largest for the Hand stimulus and the Open Hand stimulus, and not evident for the Diamond stimulus. As described next, this summary is supported by inferential statistics.

We used a multi-level modeling analysis, namely the SPSS "mixed" procedure (see Raudenbush and Bryk, 2002). Multi-level modeling is similar to least squares regression in that regressionlike parameters are estimated (e.g., the slope of the Number of beans effect). However, the parameters and their standard errors (SE) are estimated using maximum likelihood estimation instead of least squares regression. Multi-level modeling works well with within-subject designs because the maximum likelihood estimation 
obviates some typically problematic assumptions of the ANOVA such as sphericity. Also, it is particularly well-suited to the current experiment given that we wish to determine if there is a linear effect of Number of beans.

The parameters in the model corresponded to Number of beans (treated as a continuous factor with $1 \mathrm{df}$ ), Direction of bean movement (1 df), Visual Stimulus ( 2 df), and their interactions. Number of beans and Direction of bean movement were grand mean centered. Visual stimulus was coded by two, single-df orthogonal contrasts, namely the biological stimuli (Hand and Open Hand) versus the Diamond stimulus (BvsD), and Hand versus Open Hand (HvsO). In addition, a parameter was used to model total time in the threshold determination task, thus treating it as a covariate.

In the primary analysis, there were main effects of BvsD, $t(209.74)=-4.13, \mathrm{SE}=0.23$, and $\mathrm{HvsO}, t(209.74)=15.24, \mathrm{SE}=0.39$, both $p s<0.01$. More importantly, there was a significant interaction between Number of beans, Direction of bean movement, and BvsD, $t(209.74)=-2.41, \mathrm{SE}=0.002, p<0.02$. As seen in Figure 2, the interaction between number of beans and direction is visually apparent for the biological stimuli, but not for the geometrical diamond stimulus. Considering the Hand stimulus alone, the interaction between Number of beans and Direction is not significant, $p=0.09$, but there is a significant effect of Direction at 405 beans, $t(19)=2.28$, $p=0.033$, and nowhere else. Considering the Open Hand stimulus, the interaction is significant, $t(102)=-2.22, \mathrm{SE}=0.005, p=0.03$, but the effect at 405 beans is not, $t(19)=1.80, p=0.10$. Finally, for the Diamond stimulus, neither the interaction nor the effect of Direction at 405 beans was significant, $p s>0.44$. Considering the data for just the 405 bean condition, there was a significant effect of $\mathrm{HvsO}$, and importantly, a significant interaction between Direction of bean movement and BvsD, $t(43.43)=-2.54, \mathrm{SE}=0.42, p<0.02$. That is, the effect of Direction of movement was much larger for the biological stimuli than for the geometric stimulus.

\section{DISCUSSION}

The pattern of data corresponds closely to what is expected from the adaptation of a MM. First, the adaptation is cross-modal: blindfolded motor adaptation affects visual perception. Second, the effect is direction specific and consistent with the expected loss of efficiency associated with habituation mechanisms (e.g., Thompson and Burr, 2008): bean movement in the Toward direction changes the threshold so that ambiguous movement is less likely to be perceived in the Toward direction (the threshold moves so that more "toward" information is needed to perceive movement in that direction), and vice versa for Away bean movement.

Third, as expected from an adaptation mechanism, but contrary to interpretations based on task demands or expectancies, the perceptual aftereffect increases with the amount of adaptation. Nonetheless, it is surprising that there was little effect with 135 beans, especially given the effects found by Cattaneo et al. (submitted) with just 1 min of motor adaptation. We think that several factors combined to reduce statistical power. First, it is likely that any real effect at 135 beans is weak because of little opportunity for adaptation. Second, because the Direction of bean movement is manipulated between-subjects, power is lower than would be in a within-subjects design as used in Cattaneo et al. Related, there were only 15 participants in each Direction at 135 beans. Finally, the measure of the threshold is itself quite variable in part because the procedure is self-paced and hence different participants will complete the measure with different amounts of adaptation.

The fourth way in which these data suggest adaptation of a MM is that the effect is stimulus specific, namely it is strongest for perception of the biological stimuli similar to the adapted action, and not evident for the non-biological stimulus (the diamond). Note that the match between the actual action produced by the participant and the visual stimulus (e.g., a tile of hands holding small red balls) was crude, to say the least. Thus, apparently we have tapped into a MM that is more sensitive to goals than to particulars of the movement. Nonetheless, it is probably impossible to definitively attribute these effects to adaptation of a MM through behavioral means alone. Thus, as reviewed below, results from Cattaneo et al. (submitted) using TMS take on additional importance.

We did not observe a fifth hallmark of neural adaptation, namely that it is time-limited. One might expect to see a greater effect of the Direction of bean movement early in the threshold measurement than later. Unfortunately, our data are too noisy to make such a comparison definitive. In contrast, Cattaneo et al. (submitted) did observe the expected time-limited effect.

In the Cattaneo paradigm, participants used the hand to move chickpeas in a container for $60 \mathrm{~s}$. Either the palm-side of the fingers were used to pull the chickpeas in a Toward direction, or the backs of the fingers were used to push the chickpeas in an Away direction. Next, participants saw a series of pictures of a hand interacting with a ball. Each picture was displayed for $500 \mathrm{~ms}$, and for each picture the participant classified the movement as a push or a pull. Some of the pictures were completely ambiguous as to the implied direction, whereas other pictures provided information as to the direction (e.g., the ball was on the palm-side of the hand). TMS, presented simultaneously with picture onset, was also manipulated. In one condition, no TMS pulse was presented. In the other conditions, the TMS pulse was directed at motor cortex, premotor cortex, or was a sham pulse midway between the two.

Several results from the Cattaneo experiment are relevant. First, without a TMS pulse, there was a clear effect of direction of movement: After $60 \mathrm{~s}$ of pulling the chickpeas in a Toward direction, participants were less likely to classify a picture as pulling in a Toward direction; similarly, after $60 \mathrm{~s}$ of pushing the chickpeas Away, participants were less likely to classify a picture as pushing Away. This result is a conceptual replication of the results reported here. Second, when no TMS was used, the time-limited nature of the adaptation was evident. That is, the effect of direction of the $60 \mathrm{~s}$ of movement on the classification of ambiguous pictures lasted for only $30 \mathrm{~s}$. Third, the effect of direction of movement was also found for the sham TMS pulse, and it was found when the pulse was directed at motor cortex. However, the effect of direction of movement was completely eliminated when the pulse was directed at premotor cortex. Given that TMS is particularly effective on adapted systems (Cattaneo et al., 2010), this result indicates that the motor adaptation was primarily a premotor phenomenon. This finding helps to secure the claim that the adaptation paradigm is affecting a MM. That is, in the macaque, mirror neurons are more prevalent in premotor areas than motor cortex.

A possible alternative explanation for the effects shown in Figure 2 is based on visual imagery. Suppose that the blindfolded participants used repeated imagery to guide their repeated bean movements. 
Then, visual or imaginal mechanisms might adapt, implying that the effects in Figure 2 are not cross-modal at all. A related alternative involves back-projections from motor and spatial-attention areas to visual areas. Repeated motor actions would, by these routes, stimulate visual areas, possibly producing adaptation without any form of consciously controlled visual imagery. Therefore, the adapted units need not be a motor-based MM.

These alternatives are unlikely for four reasons. First, introspection during the bean task suggests that there is no need for imagery. Second, recall that participants kept their left hands on the target container. Thus guidance of bean movement is more likely based on proprioception than visual imagery. Third, Dinstein et al. (2008) report repetition suppression of the BOLD signal with both observed action and blindfolded produced action with no need for visual imagery in the later case. Fourth, these accounts are at least partially vitiated by the Cattaneo et al. (submitted) TMS data. Using TMS, Cattaneo demonstrated that the motor adaptation task affects premotor areas. Consequently, it is unlikely that the observed adaptation is solely due to visual mechanisms.

Nonetheless, it is possible that the motor adaptation also produces an effect on visual processing. Consider, for example, the Damasio and Meyer (2008) interpretation of MMs. According to these authors, MMs may be similar to convergence zones that bring together activity in diverse areas of cortex. On this account, adapting a MM would also affect processing in visual areas through changes in "retro-activation" from the MM.

Our results are consistent with several other reports of human MM systems using primarily behavioral techniques that focus on adaptation (or learning). For example, Catmur et al. (2007) measured muscle evoked potentials (MEPs) using TMS while participants watched a video of a hand moving either the index finger or little finger. As expected, there was a congruency effect: MEPs were largest in the observer's muscle corresponding to that used to move the finger in the video. Next, Catmur et al. (2007) had participants practice either the congruent movement (e.g., move the index finger upon seeing the index finger move) or an incongruent movement (e.g., move the little finger upon seeing the index finger move). After training, TMS was again used to measure MEPs associated with the videos. The major result was that the incongruent training reversed the congruency effect observed in the MEPs. Whereas these results are consistent with the MM hypothesis, they do not demonstrate that the system tapped by training was used in action recognition. That is, the MEPs might index activation of motor system during action observation, but the MEPs do not indicate that the motor system plays a causal role in action recognition.

Casile and Giese (2006) do show a causal effect of motor training on action recognition. In their research, blindfolded participants were asked to make novel upper body movements. Later, participants were better able to recognize point-light displays that incorporated the trained movement. Importantly, and similar to our dose-response effect of number of beans moved, Casile and Giese demonstrated that degree of learning the novel movements correlated with recognition of the point-light displays. These results are consistent with a human MM explanation. Nevertheless, because Casile and Giese do not show biological specificity (e.g., that the effect is not found for the recognition of non-biological movement) the results are not as convincing as they might be.
Our results appear to contrast with those of Casile and Giese. Casile and Giese show a facilitation effect of motor training (better recognition of a particular gait in point-light displays), whereas we show a type of inhibition (more information needed to see movement in a particular direction). A likely reason for this difference is that Casile and Giese trained their participants with novel movements for which it is unlikely that any MM had previously developed. Thus, any training is more likely to help than hinder performance. Our participants practiced a well-learned action. In this case, the practice might produce benefits, or for several reasons, the adaptation might appear to be inhibitory. For example, the repeated bean movement using a particular grip with a particular size bean and a particular direction and extent might drive the system toward a specialization for these components of the action (cf. Catmur et al., 2007). Then, when the MM is presented with the not-quite-identical visual stimuli in the threshold measurement task, the increased specialization hinders recognition using the MM.

The behavioral adaptation paradigm provides several benefits. As noted, causal hypotheses can be tested, specificity for recognition of biological action can be found, and there is clear evidence of physiological adaptation (the effect of number of beans). In addition, the adaptation paradigm provides a general method for studying function of MMs. For example, bean movement adaptation also affects sentence comprehension (Glenberg et al., 2008). In that research, following movement of 600 beans in the Toward or Away direction, participants judged the sensibility of sentences describing transfer toward the reader (e.g., "Mike gives you the pen") or away (e.g., "You give Mike the pen"). Following Toward bean movement, participants were slowed in their judgment of sentences describing transfer toward (and vice versa for Away bean movement). This finding is consistent with the claims that (a) understanding sentences describing action requires a simulation of that action using the motor system (Glenberg and Kaschak, 2002) and (b) this simulation taps MMs (Rizzolatti and Craighero, 2007). In addition, note that the direction of the effect is consistent with that in Figure 2. That is, adapting toward-action control slows understanding of descriptions of action toward the reader.

A second example of the generality of the motor adaptation procedure is provided by Sato et al. (2008). Participants differentially adapted speech articulators by either pursing the lips 150 times or lifting the tongue to behind the teeth 150 times. Following adaptation, the participants identified syllables as "pa" or "ta." As measured by the signal detection parameter beta, lip adaptation biased classification of stimuli as "pa," whereas tongue adaptation biased classification as "ta".

The cross-modal adaptation paradigm holds promise to be extended in several directions. For example, if MMs are sensitive to social information, then observing another person move beans should adapt the observer's MM (see Havas et al., 2010). Additionally, the motor task can be modified to study the learning of MMs, changes across the lifespan, and the relation between MMs and various psychological syndromes.

In conclusion, we have demonstrated the use of a behavioral technique for investigating human MMs through cross-modal adaptation. We cannot be positive that we are tapping into the 
same sort of MM revealed using single-cell recording. However, the technique produces data that are very close to what would be expected it that were the case, namely, a cross-modal effect that depends on the amount of adaptation and that shows some degree of biological specificity. Thus we think that this paradigm will become a useful means for investigating the causal contributions of MMs to cognition.

\section{REFERENCES}

Calvo-Merino, B., Grezes, J., Glaser, D. E., Passingham, R. E., and Haggard, P. (2006). Seeing or doing? Influence of visual and motor familiarity in action observation. Curr. Biol. 16, 1905-1910.

Casile, A., and Giese, M. A. (2006). Nonvisual motor training influences biological motion perception. Curr. Biol. 16, 69-74.

Catmur, C., Walsh, V., and Heyes, C. (2007). Sensorimotor learning configures the human mirror system. Curr. Biol. 17, 1527-1531.

Cattaneo, L., Sandrini, M., and Schwarzbach, J. (2010). Statedependent TMS reveals a hierarchical representation of observed acts in the temporal, parietal, and premotor cortices. Cereb. Cortex. doi: bhp291[pii]10.1093/cercor/bhp291.

Chong, T. T.-J., Cunnington, R., Williams, M. A., Kanwisher, N., and Mattingley, J. B. (2008). FMRI adaptation reveals mirror neurons in human inferior parietal cortex. Curr. Biol. 18, 1576-1580.

Classen, J., Liepert, J., Wise, S. P., Hallett, M., and Cohen, L. G. (1998). Rapid plasticity of human cortical movement representation induced by practice. $J$. Neurophysiol. 79, 1117-1123.

Damasio, A., and Meyer, K. (2008). Behind the looking-glass. Nature 454, 167-168.

Dapretto, M., Davies, M. S., Pfeifer, J. H., Scott, A. A., Sigman, M., Bookheimer, S. Y., and Iacoboni, M. (2006). Understanding emotions in others: mirror neuron dysfunction in children with autism spectrum disorders. Nat. Neurosci. 9, 28-30.

D’Ausilio, A., Pulvermuller, F., Salmas, P., Buflari, I., Begliomini, C., and Fadiga, L. (2009). The motor somatotopy of speech perception. Curr. Biol. 19, 381-385.
Dinstein, I. (2008). Human cortex: reflections of mirror neurons. Curr. Biol. 18, R956-R959.

Dinstein, I., Thomas, C., Behrmann, M., and Heeger, D. J. (2008). A mirror up to nature. Curr. Biol. 18, R13-R18.

Di Pellegrino, G., Fadiga, L., Fogassi, L., Gallese, V., Rizzolatti, G. (1992). Understanding motor events: a neurophysiological study. Exp. Brain Res. 91, 176-180.

Gazzola,V.,Aziz-Zadeh, L., and Keysers, C. (2006). Empathy and the somatotopic auditory mirror system in humans. Curr. Biol. 16, 1824-1829.

Glenberg, A. M. (2007). "Language and action: creating sensible combinations of ideas," in The Oxford Handbook of Psycholinguistics, eds G. Gaskell (Oxford, UK: Oxford University Press), 361-370.

Glenberg, A. M., and Kaschak, M. P. (2002). Grounding language in action. Psychon. Bull. Rev. 9, 558-565.

Glenberg, A. M., Sato, M., and Cattaneo, L. (2008). Use-induced motor plasticity affects the processing of abstract and concrete language. Curr. Biol. 18,

Havas, D., Jenvey, J., Shilling, H., and Nathan, M. (2010). "Socially induced motor plasticity affects language comprehension," Paper Presented at the Meeting of the Cognitive Science Society, Portland, OR.

Hickok, G. (2009). Eight problems for the mirror neuron theory of action understanding in monkeys and humans. J. Cogn. Neurosci. 21, 1229-1243.

Kilner, J. M., Marchant, J. L., and Frith, C. D. (2006). Modulation of the mirror system by social relevance. Soc. $\operatorname{Cog} n$. Affect. Neurosci. 1, 143-148.

Kilner, J. M., Neal, A., Weiskopf, N., and Frith, C. D. (2009). Evidence of mirror neurons in human inferior frontal gyrus. J. Neursoci. 29, 10153-10159. R290-R291.

\section{ACKNOWLEDGMENTS}

We thank the reviewers whose comments prompted improvements in this report. Work on this article was supported by the National Science Foundation under Grant No. BCS 0744105 to Arthur Glenberg. Any opinions, findings, and conclusions or recommendations expressed in this material are those of the authors and do not necessarily reflect the views of the National Science Foundation.

Kohler, E., Keysers, C., Umiltà, M. A., Fogassi, L., Gallese, V., and Rizzolatti, G. (2002). Hearing sounds, understanding actions: action representation in mirror neurons. Science 297, 846-848.

Lewis, C. F., and McBeath, M. K. (2004) Bias to experience approaching motion in a three-dimensional virtual environment. Perception 33, 259-276.

Lingnau, A., Gesierich, B., and Caramazza A. (2009). Asymmetric fMRI adaptation reveals no evidence for mirror neurons in humans. Proc. Natl. Acad. Sci. 106, 9925-9930.

Mukamel, R., Ekstrom, A. D., Kaplan, J., Iacoboni, M., and Fried, I. (2010) Single-neuron responses in humans during execution and observation of actions. Curr. Biol. doi: 10.1016/j. cub.2010.02.045.

Raudenbush, S. W., and Bryk, A. S. (2002). Hierarchical Linear Models: Applications and Data Analysis Methods. Thousand Oaks, CA: Sage Publications.

Rizzolatti, G., and Craighero, L. (2004). The mirror-neuron system. Annu. Rev. Neurosci. 27, 169-192.

Rizzolatti, G., and Craighero, L. (2007) "Language and mirror neurons," in The Oxford Handbook of Psycholinguistics eds G. Gaskell (Oxford, UK: Oxford University Press), 771-786.

Sato, M., Brisebois, A., Grabski, K. Basirat, A., Ménard, L., Glenberg, A. M., and Cattaneo, L. (2008). Paperpresented at the Speech and Face to Face Communication Workshop, Grenoble, France.

Tettamanti M., Buccino G., Saccuman M. C., Gallese V., Danna M., Scifo, P., Fazio, F., Rizzolatti, G., Cappa S. F., and Perani, D. (2005). Listening to action-related sentences activates fronto-parietal motor circuits. J. Cogn. Neurosci. 17, 273-281.
Thompson, P., and Burr, D. (2008). Visual aftereffects. Curr. Biol. 19, R11-R14.

Tyrrell, R. A., and Owens, D. A. (1988). A rapid technique to assess the resting states of the eyes and other threshold phenomena - the modified binary search (MOBS). Behav. Res. Methods Instrum. Comput. 20, 137-141.

Umiltà, M. A., Kohler, E., Gallese, V., Fogassi, L., Fadiga, L., Keysers, C., and Rizzolatti, G. (2008). When pliers become fingers in the monkey motor system. Proc. Natl. Acad. Sci. 105, 2209-2213.

Umiltà, M.A., Kohler, E., Gallese, V., Fogassi, L., Fadiga, L., Keysers, C., and Rizzolatti, G. (2001). I know what you are doing: a neurophysiological study. Neuron 31, 155-165.

Conflict of Interest Statement: The authors declare that the research was conducted in the absence of any commercial or financial relationships that could be construed as a potential conflict of interest.

Received: 27 May 2010; accepted: 07 October 2010; published online: 04 November 2010.

Citation: Glenberg AM, Lopez-Mobilia G, McBeath M, Toma $M$, Sato $M$ and Cattaneo L (2010) Knowing beans: human mirror mechanisms revealed through motor adaptation. Front. Hum. Neurosci. 4:206. doi: 10.3389/ fnhum.2010.00206

Copyright $(2010$ Glenberg, Lopez-Mobilia, McBeath, Toma, Sato and Cattaneo. This is an open-access article subject to an exclusive license agreement between the authors and the Frontiers Research Foundation, which permits unrestricted use, distribution, and reproduction in any medium, provided the original authors and source are credited. 